\title{
Efficient maternal to neonatal transfer of antibodies against SARS-CoV-2 and BNT162b2 mRNA COVID-19 vaccine
}

Ofer Beharier, ${ }^{1,2}$ Romina Plitman Mayo, ${ }^{2}$ Tal Raz, ${ }^{3}$ Kira Nahum Sacks, ${ }^{4}$ Letizia Schreiber, ${ }^{5}$ Yael Suissa-Cohen, ${ }^{1}$ Rony Chen, ${ }^{6}$ Rachel Gomez-Tolub, ${ }^{6}$ Eran Hadar, ${ }^{6}$ Rinat Gabbay-Benziv, ${ }^{7}$ Yuval Jaffe Moshkovich, ${ }^{7}$ Tal Biron-Shental, ${ }^{8}$ Gil Shechter-Maor, ${ }^{8}$ Sivan Farladansky-Gershnabel, ${ }^{8}$ Hen Yitzhak Sela, ${ }^{9}$ Hedi Benyamini-Raischer, ${ }^{10}$ Nitzan D. Sela, ${ }^{10}$ Debra Goldman-Wohl, ${ }^{1}$ Ziv Shulman, ${ }^{11}$ Ariel Many, ${ }^{12}$ Haim Barr, ${ }^{13}$ Simcha Yagel, ${ }^{1}$ Michal Neeman, ${ }^{2}$ and Michal Kovo ${ }^{4}$

${ }^{1}$ Department of Obstetrics and Gynecology, Hadassah-Hebrew University Medical Center, Jerusalem, Israel. ${ }^{2}$ Department of Biological Regulation, Weizmann Institute of Science, Rehovot, Israel. ${ }^{3}$ Koret School of Veterinary Medicine, The Robert H. Smith Faculty of Agriculture, Food \& Environment, The Hebrew University of Jerusalem, Rehovot, Israel. ${ }^{4}$ Department Obstetrics and Gynecology, Wolfson Medical Center, Holon; affiliated to Sackler Faculty of Medicine, Tel Aviv University, Tel Aviv, Israel. ${ }^{5}$ Department of Pathology, Wolfson Medical Center, Holon; affiliated to Sackler Faculty of Medicine, Tel Aviv University, Tel Aviv, Israel. ${ }^{6}$ Helen Schneider Hospital for Women, Rabin Medical Center, Petach Tikva; affiliated to Sackler Faculty of Medicine, Tel Aviv University, Israel. ${ }^{7}$ The Hillel Yaffe Medical Center, Hadera, Israel; affiliated to the Bruce Rappaport Faculty of Medicine, Technion, Israel Institute of Technology, Haifa, Israel. ${ }^{8}$ Department of Obstetrics and Gynecology, Meir Medical Center, Kfar Saba, Israel, affiliated to Sackler School of Medicine, Tel Aviv University, Tel Aviv, Israel. ${ }^{9}$ Department of Obstetrics and Gynecology, Shaare Zedek Medical Center and Faculty of Medicine, Hebrew University of Jerusalem, Israel. ${ }^{10}$ Department of Obstetrics and Gynecology, Emek Medical Center, Afula, Israel affiliated with Rappaport Faculty of Medicine, Technion, Haifa, Israel. ${ }^{11}$ Department of Immunology, Weizmann Institute of Science, Rehovot, Israel. ${ }^{2}$ Lis Hospital for Women, Tel Aviv Sourasky Medical Center, Tel Aviv, Israel; affiliated to Sackler Faculty of Medicine, Tel Aviv University, Tel Aviv, Israel. ${ }^{13}$ The Nancy and Stephen Grand Israel National Center for Personalized Medicine (G-INCPM), Weizmann Institute of Science, Rehovot, Israel.

BACKCROUND. The significant risks posed to mothers and fetuses by COVID-19 in pregnancy have sparked a worldwide debate surrounding the pros and cons of antenatal SARS-CoV-2 inoculation, as we lack sufficient evidence regarding vaccine effectiveness in pregnant women and their offspring. We aimed to provide substantial evidence for the effect of the BNT162b2 mRNA vaccine versus native infection on maternal humoral, as well as transplacentally acquired fetal immune response, potentially providing newborn protection.

METHODS. A multicenter study where parturients presenting for delivery were recruited at 8 medical centers across Israel and assigned to 3 study groups: vaccinated $(n=86)$; PCR-confirmed SARS-CoV-2 infected during pregnancy $(n=65)$, and unvaccinated noninfected controls $(n=62)$. Maternal and fetal blood samples were collected from parturients prior to delivery and from the umbilical cord following delivery, respectively. Sera IgC and IgM titers were measured using the Milliplex MAP SARS-CoV-2 Antigen Panel (for S1, S2, RBD, and N).

RESULTS. The BNT162b2 mRNA vaccine elicits strong maternal humoral IgG response (anti-S and RBD) that crosses the placenta barrier and approaches maternal titers in the fetus within 15 days following the first dose. Maternal to neonatal antiCOVID-19 antibodies ratio did not differ when comparing sensitization (vaccine vs. infection). IgG transfer ratio at birth was significantly lower for third-trimester as compared with second trimester infection. Lastly, fetal IgM response was detected in 5 neonates, all in the infected group.

CONCLUSION. Antenatal BNT162b2 mRNA vaccination induces a robust maternal humoral response that effectively transfers to the fetus, supporting the role of vaccination during pregnancy.

FUNDING. Israel Science Foundation and the Weizmann Institute Fondazione Henry Krenter.

\section{Introduction}

The worldwide pandemic of COVID-19 continues to spread, with substantial morbidity and mortality. To date, more than

Authorship note: $\mathrm{OB}$ and RPM contributed equally to this study. Conflict of interest: The authors have declared that no conflict of interest exists. Copyright: (5) 2021, American Society for Clinical Investigation.

Submitted: April 7, 2021; Accepted: May 19, 2021; Published: May 20, 2021.

Reference information: J Clin Invest. 2021;131(13):e150319.

https://doi.org/10.1172/JCl150319.
80,000 pregnant women have been infected in the U.S. alone, and the estimated global number of pregnant women infected with COVID-19 is likely to reach millions this year. Recent data demonstrated that pregnant women with COVID-19 infection are at increased risk for intensive care unit (ICU) admission, mechanical ventilation, and death, compared with properly matched nonpregnant women (1-9). Furthermore, COVID-19 illness increases the risk for pregnancy complications such as preterm birth, pregnancy-induced hypertensive diseases, and thromboembolic diseases (10). Although accumulating data suggest that the risk for 
Table 1. Clinical parameters of women included in the study

\begin{tabular}{|c|c|c|c|}
\hline Parameter & $\begin{array}{c}\text { Control group } \\
n=66\end{array}$ & $\begin{array}{l}\text { Past SARS-CoV-2 group } \\
\qquad n=74\end{array}$ & $\begin{array}{c}\text { Vaccinated group } \\
\quad n=92\end{array}$ \\
\hline Maternal age, mean $\pm S D$, years & $31.6 \pm 5.8$ & $28.8 \pm 5.8^{A}$ & $31.7 \pm 5.8$ \\
\hline Gestational age, mean \pm SD, weeks & $39.2 \pm 1.4$ & $39 \pm 1.6$ & $39.3 \pm 1.3$ \\
\hline Preterm delivery (<37), $n(\%)$ & $5(7.6)$ & $8(10.8)$ & $4(4.3)$ \\
\hline Pregravid BMI $\left(\mathrm{kg} / \mathrm{m}^{2}\right)$, mean $\pm \mathrm{SD}$ & $25.7 \pm 6.5$ & $26.4 \pm 9.2$ & $24.2 \pm 5.2$ \\
\hline Gravidity, median (IQR) & $3(2.5)$ & $3(2)$ & $3(2)$ \\
\hline Parity, median (IQR) & $2(2)$ & $1(3)$ & $1(2)$ \\
\hline \multicolumn{4}{|l|}{ Maternal comorbidities, $n(\%)$} \\
\hline Hypertensive disorders & $1(1.5)$ & $1(1.4)$ & $1(1.1)$ \\
\hline Diabetes or gestational diabetes & $9(13.6)$ & $4(5.4)$ & $8(8.7)$ \\
\hline Asthma & $1(1.5)$ & $2(2.7)$ & $2(2.2)$ \\
\hline Thyroid disease & $4(6.1)$ & $1(1.3)$ & $8(8.6)$ \\
\hline Smoker & $4(6.6)$ & $1(1.4)$ & $6(6.5)$ \\
\hline \multicolumn{4}{|l|}{ Infant sex, $n(\%)$} \\
\hline Male & $31(47.7)$ & $39(52)$ & $45(49.5)$ \\
\hline Female & $34(52.3)$ & $36(48)$ & $46(50.5)$ \\
\hline Birthweight, mean $\pm S D$, grams & $3239.4 \pm 395.7$ & $3324.1 \pm 536.2$ & $3281.8 \pm 420.2$ \\
\hline NICU, $n(\%)$ & $1(1.6)$ & $2(2.7)$ & $4(4.3)$ \\
\hline PCR-positivity GA, mean \pm SD, weeks & - & $28.1 \pm 8.3$ & - \\
\hline First vaccine dose $\mathrm{GA}$, mean $\pm \mathrm{SD}$, weeks & - & - & $34.5 \pm 7.5$ \\
\hline
\end{tabular}

Continuous parameters were analyzed by Kruskal-Wallis 1-way ANOVA test, following by Dunn's all-pairwise comparisons test; Pearson $\chi^{2}$ analysis was used to compare proportional data. ${ }^{A}$ Clinical parameters did not differ among the groups, except for maternal age, which was significantly lower in the SARS-CoV-2 group, as compared with the other 2 groups (Kruskal-Wallis 1-way ANOVA; $P=0.0011$ ) tion campaign among pregnant women in Israel began in December 2020. Herein, our objective was to evaluate the maternal production and placental transfer of antibodies following vaccination with the BNT162b2 mRNA vaccine and natural SARS-CoV-2 infection during pregnancy. We analyzed BNT162b2 mRNA vaccineinduced IgG and IgM antibody concentrations in maternal and cord blood samples from 105 deliveries at 8 medical centers in Israel between January and March 2021. Furthermore, we compared these results to IgG and IgM antibody concentrations in maternal and cord blood samples from 74 deliveries of women with PCR confirmed SARS-CoV-2 infection, contracted during various stages of pregnancy, as well as to 62 noninfected unvaccinated matched pregnant controls collected between April 2020 and March 2021.

\section{Results}

Participant characteristics. The cohort consisted of 1094 participants from 8 hospitals across Israel. Samples were collected between April 2020 and March 2021 following childbirth and stratified into 3 groups: 105 vaccine recipients (all collectsevere morbidity and mortality among infected pregnant women is low, the Centers for Disease Control and Prevention (CDC) (11) included pregnancy as a risk factor for severe COVID-19 illness, a statement that was also acknowledged by the American College of Obstetricians and Gynecologists (ACOG), the Society for Maternal-Fetal Medicine (SMFM), and other women's health organizations $(10,12,13)$.

An important aspect of the maternal response to COVID-19 infection is the rapid resolution of infection by neutralizing immune response and transfer of immunity to the newborn. The maternal immune system plays a unique role in pregnancy, since the newborn depends on the active transfer of maternal immunoglobulin $G(\operatorname{IgG})$ across the placenta for its protection against pathogens (14-16). Recent data revealed decreased placental transfer of COVID-19-specific antibodies (17), secondary to altered glycosylation profile $(17,18)$. Proper transfer of neutralizing antibodies may be critical during pregnancy, as a greater proportion of neonates and infants have severe or critical illness upon COVID-19 infection than older pediatric counterparts $(19,20)$.

In an attempt to stop the COVID-19 pandemic spread, mass vaccination campaigns commenced worldwide. Randomized clinical trials reported efficacy of $94 \%$ (21) to $95 \%$ (22) for mRNAbased vaccines; however, these studies excluded pregnant women. Nevertheless, following extensive discussion regarding the risk of COVID-19 during pregnancy, potential vaccine benefits, and safety concerns, the CDC, the Israel Ministry of Health, and other health organizations advised that the COVID-19 vaccine should be offered to pregnant women. Accordingly, a vaccina- ed between January to March 2021), 94 unvaccinated participants with past SARS-CoV-2-positive RT-PCR results, and 895 unvaccinated participants without prior documentation for infection (Table 1 and Figure 1). Matched maternal cord blood serology results were obtained for 213 dyads; 65 with past RT-PCR-positive results, 86 vaccinated recipients ( 3 of whom were also PCR-positive during pregnancy), and 62 noninfected unvaccinated individuals; 55 enrolled participants did not have matched cord blood serological results for analysis and were therefore excluded from the analyses. Among the 895 noninfected unvaccinated participants, 66 were selected (based on clinical parameters) as a matched comparison group, and 4 were later excluded due to lack of matched maternal cord blood serology results. Participant demographic and clinical characteristics and outcomes are provided by study groups in Table 1 . Briefly, the participants were pregnant women from all ethnic societal groups in Israel ( 75\% Jewish, $\sim 20 \%$ Arab, $5 \%$ other). Diversity was enhanced by the large number of medical centers included in this study, servicing all communities. Clinical parameters did not differ among the groups, except for maternal age, which was significantly lower in the SARS-CoV-2 group, as compared with the other 2 groups (Kruskal-Wallis 1-way ANOVA; $P=0.0011$ ).

Maternal and fetal serological response to SARS-CoV-2 infection. Maternal (Figure 2A) and cord (Figure 2B) blood serological IgG response to $\mathrm{S} 1, \mathrm{~S} 2, \mathrm{RBD}$, and $\mathrm{N}$ antigens of SARS-CoV-2 were derived at the time of delivery from 65 patients who were PCR positive during pregnancy. The data were analyzed by the gestational age (GA) of PCR diagnosis to align all patients on a common 


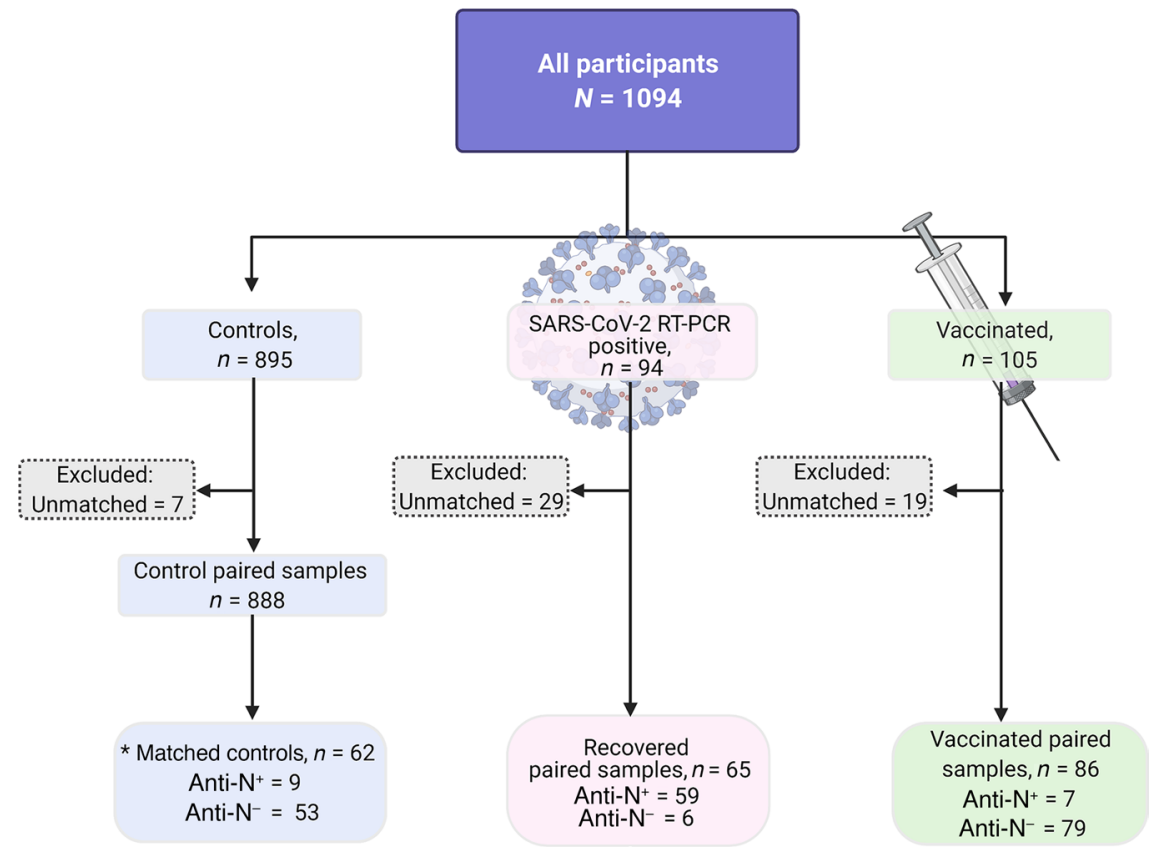

Figure 1. Patient selection flow chart. Patients were recruited from 8 medical centers in Israel and all were SARS-CoV-2 RT-PCR negative at delivery. Seropositivity for nucleocapsid (N) was set at the level of the top $90 \%$ of the PCR-positive recovered group, and verified by positivity for $\mathrm{S1}, \mathrm{S2}$, and RBD (see Supplemental Figures 1 and 2). The same threshold was used to reveal seropositive cases in the vaccinated and the control groups. gestational time axis to reveal possible changes in the humoral response or infection biology across pregnancy.

Based on serology analyses at delivery (Figure 2), transmission rates of IgG to S1, S2, RBD, and N antigens were significantly higher in participants who were PCR positive to SARS-CoV-2 prior to gestational week $30(n=25)$, as compared with gestational week $>30(n=21)$ (Wilcoxon rank sum test. $\mathrm{S} 1, P=0.0013$; S2, $P=$ 0.0231 ; RBD $P=0.0010 ; \mathrm{N}, P=0.0003)$. Maternal to fetal transfer ratio was defined as fetal divided by maternal antibody levels:

$$
T R=\frac{\text { Fetal- } \lg G(\mathrm{MFI})}{\text { Maternal-IgG(MFI) }}
$$

Equation 1

TR is the transfer ratio, and MFI is the mean fluorescence intensity. Maternal to fetal IgG TR values were consistently below 1 for infection occurring at GA greater than 30 weeks, but were significantly elevated at delivery for infections prior to GA 30 (Pearson's $\chi^{2}, P<0.0001$; Figure $2 \mathrm{C}$ ).

Serological based reclustering of the study groups. Based on the robust serological response maintained from midpregnancy PCR verified SARS-CoV-2 infection until delivery, the multiplexed immune response was used for further clustering of the participants based on the reactivity to the $\mathrm{N}$ antigen. Multiplexed serological IgG and IgM response to S1, S2, RBD, and N were tested in maternal and neonate sera (Supplemental Figure 1; supplemental material available online with this article; https://doi.org/10.1172/ JCI150319DS1). In particular, the response to $\mathrm{N}$ (anti-N, present in SARS-CoV-2 but not in the BNT162b2 mRNA vaccine) versus $\mathrm{RBD}$ (anti-RBD, present in both SARS-CoV-2 and the BNT162b2 mRNA vaccine) separated the main groups and identified additional potentially infected participants of the vaccinated and control groups (Supplemental Figure 2). Among 65 participants with past SARS-CoV-2 RT-PCR-positive test, the top $90 \%$ of the mater- nal IgG response for $\mathrm{N}$ was defined as seropositive (IgG $\mathrm{N}$ [MFI] $>1583 ; n=59)$. Within the control group, 9 participants $(14 \%)$ were seropositive for $\mathrm{N}$ using the above threshold, which together with IgG seropositivity to the other COVID-19 antigens (S1, S2, and RBD), corresponds to a preexisting induced immunity due to infection. Similarly, 7 vaccinated participants $(8 \%)$ were seropositive for $\mathrm{N}$, of which 3 were also PCR-positive.

Notably, within the PCR-positive group, 4 neonates were identified with robust IgM response to all SARS-CoV-2 antigens, and an additional neonate showed partial response consistent with compromised placenta barrier, fetal exposure to viral antigens, or with vertical viral transmission. Clinical review of these cases showed that the mothers were diagnosed with mild SARS-CoV-2 infection that spontaneously resolved weeks prior to childbirth. Three cases delivered at term, and one case gave birth at 35 weeks following preterm premature rupture of the fetal membranes. In all cases, both mother and newborn did not show any signs of illness after childbirth.

Maternal and fetal serological response to BNT162 2 vaccine. The temporal dependence of the acute maternal response to SARSCoV-2 infection (days 1-45; Figure 3A) was compared with the response to the 2-dose regime of BNT162b2; where the first vaccine dose is administered on day 1 and the second dose on day 21 (Figure 3B). A gradual rise in IgG humoral response (anti-S1, -S2, $-\mathrm{RBD}$, and $-\mathrm{N}$ ) was detected during the first 45 days after infection (Figure $3 \mathrm{~A}$ ). In the same period, vaccinated participants who received the first BNT162b2 dose showed a rapid IgG response to S1, S2, and RBD but not N, resulting in high titer values by day 15 after the first dose. A further rise in IgG was observed following the second dose (Figure 3B). The temporal dependence of fetal IgG for S1, S2, and RBD after vaccination trailed after the maternal IgG showing a marked response already by day 15 . As expected, a further increase was observed following the second vaccination dose (Figure 3C). As illustrated in Supplemental Figure 3, at the time of delivery, maternal IgG for S1 and RBD were significantly 

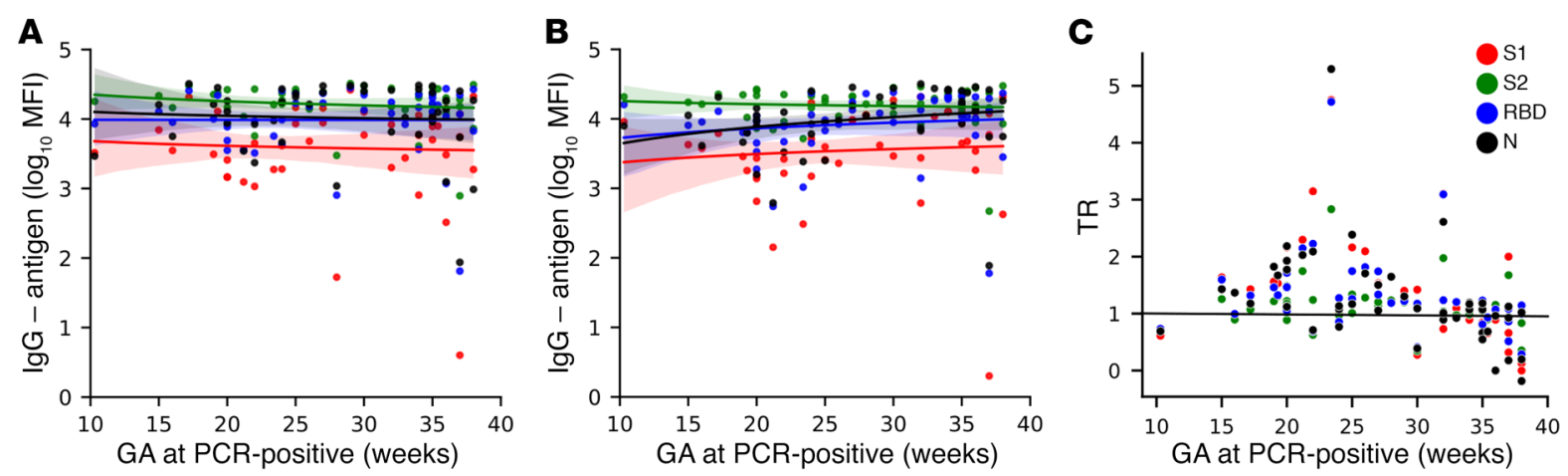

Figure 2. Robust maternal and fetal seropositivity can be detected at birth after recovery from SARS-CoV-2 infection. Maternal and cord blood were derived at the time of delivery from patients $(n=65)$ who recovered from infection with SARS-CoV-2 verified by RT-PCR at the specified CA during pregnancy. Maternal and fetal seropositivity (A and B) and transfer ratio (TR) (C) were analyzed for 4 SARS-CoV-2 antigens (S1, S2, RBD, and N). The data are plotted as a function of the GA at which positive RT-PCR was diagnosed. (A and B) High levels of maternal (A) and fetal (B) IgG levels for S1, S2, RBD, and $\mathrm{N}$ are found at delivery for infections occurring prior to GA 30, and appear to be lower for late third trimester infections. Shaded areas show the $95 \%$ confidence interval. (C) TR values at delivery are low for third trimester infections, and significantly higher for earlier, second trimester infections (Wilcoxon rank sum test, $P<0.0001$ ). S1, red; S2, green, RBD, blue; N, black. Black line marks TR $=1$.

higher in vaccinated women $(P=0.0009, P=0.0045$, respectively), while IgG for $\mathrm{S} 2$ and $\mathrm{N}$ were significantly higher in PCR-positive women $(P=0.0016, P<0.0001$, respectively). Fetal IgG for S2 and $\mathrm{N}$ were significantly lower in cord blood samples of vaccinated women $(P<0.0001, P<0.0001$, respectively), while fetal IgG for $\mathrm{S} 1$ and RBD did not differ from those of PCR-positive women $(P=0.7017, P=0.6887$, respectively).

Paired maternal-neonate serological data were grouped for statistical analysis to control unvaccinated mothers, as well as to mothers who presented at delivery within the first 3 weeks after the first vaccine dose; deliveries during the first week after the second vaccine dose; and fully vaccinated deliveries more than 1 week after the second vaccine. Significant increase in maternal and fetal $\operatorname{IgG}(P<0.0001)$ and maternal $\operatorname{IgM}(P<0.05)$ to S1, S2, and RBD but not $\mathrm{N}$ were observed already after the first vaccination dose and persisted at later time points (Supplemental Table 1). Fetal IgM response to BNT162b2 antigens (S1, S2, RBD) was negligible, consistent with no evidence for direct exposure of the fetus to vaccine-derived antigens (Figure 3D and Supplemental Figure 1).

Correlation of maternal-fetal IgG response to SARS-CoV-2 infection and vaccination. The serological response in cord blood correlated positively with the maternal humoral response for IgG against all the analyzed antigens (Figure 4A IgG-S1; Figure 4B IgG-S2; Figure 4C IgG-RBD; Figure 4D IgG-N). There were no differences between the correlation slopes of the SARS-CoV-2infected group vs. the vaccinated group for any type of antibodies (S1, $P=0.2936 ;$ S2, $P=0.4212 ; \mathrm{RBD}, P=0.09702 ; \mathrm{N}, P=0.7616$ ), suggesting similar placental antibody transfers following SARSCoV-2 infection and vaccination.

Maternal to fetal IgG transfer ratio for $S 1, S 2, R B D$, and $N$. The IgG transfer ratio was derived for the PCR-positive group and for serologically positive and negative vaccinated groups $\left(\mathrm{N}^{+}\right.$ and $\mathrm{N}^{-}$; Figure 5). Note that the TR for $\mathrm{N}$ in the $\mathrm{N}^{-}$group is not presented due to the low seropositivity. Significant differences were found for S1 (Figure 5A), S2 (Figure 5B), and RBD (Figure $5 C$ ), but not for $\mathrm{N}$ (Figure 5D) between the PCR-positive and vaccinated anti- $\mathrm{N}^{-}$groups $(P<0.0002)$. The transfer ratios for all antibodies did not differ between the vaccinated anti- $\mathrm{N}^{+}$and all the other groups $(P=0.4577)$.

\section{Discussion}

Pregnant women and their neonates are considered vulnerable populations for COVID-19 infection, with significantly greater risks for morbidity and mortality, when compared with matched populations (17). Recent studies reported that among patients infected during the third trimester, the transfer of anti-SARS-CoV-2 antibodies to the fetus is significantly impaired $(17,18)$. Indeed, our study confirmed the low transfer ratio for infections late in pregnancy. However, with the availability of a large cohort of patients infected earlier in pregnancy (weeks 15-30), we were able to show for the first time that maternal and cord blood anti-COVID-19 antibodies, generated in response to a second trimester infection, as well as transfer ratio, were high at the time of delivery in participants recovering from SARS-CoV-2 infection contracted months prior to childbirth. The low transfer ratio for infections in late gestation could thus also reflect a lag in antibody transfer across the placenta.

Following reports of a decline in antibody titers months after infection, health organizations recently recommended vaccination following natural SARS-CoV-2 infection for boosting immunity (23). However, the significance and relevance of this policy during pregnancy are the subjects of some debate and has not been supported by evidence. Based on our finding of persistent humoral immunity for infections contracted during the second trimester of pregnancy, titer testing may be informative prior to boosting previously infected pregnant woman, unless boosting is warranted by emerging variants (24). Unfortunately, pregnant women were excluded from previous clinical vaccine studies. However, the significant risks and pressing need for action led to a worldwide debate concerning SARS-CoV-2 inoculation during pregnancy, while data were still lacking. In the present study, we drew on the unprecedented vaccination campaign undertaken in Israel, which included pregnant women, and report on the robust humoral immune response following antenatal immunization with the mRNA vaccine. We found that the Pfizer-BioNTech 

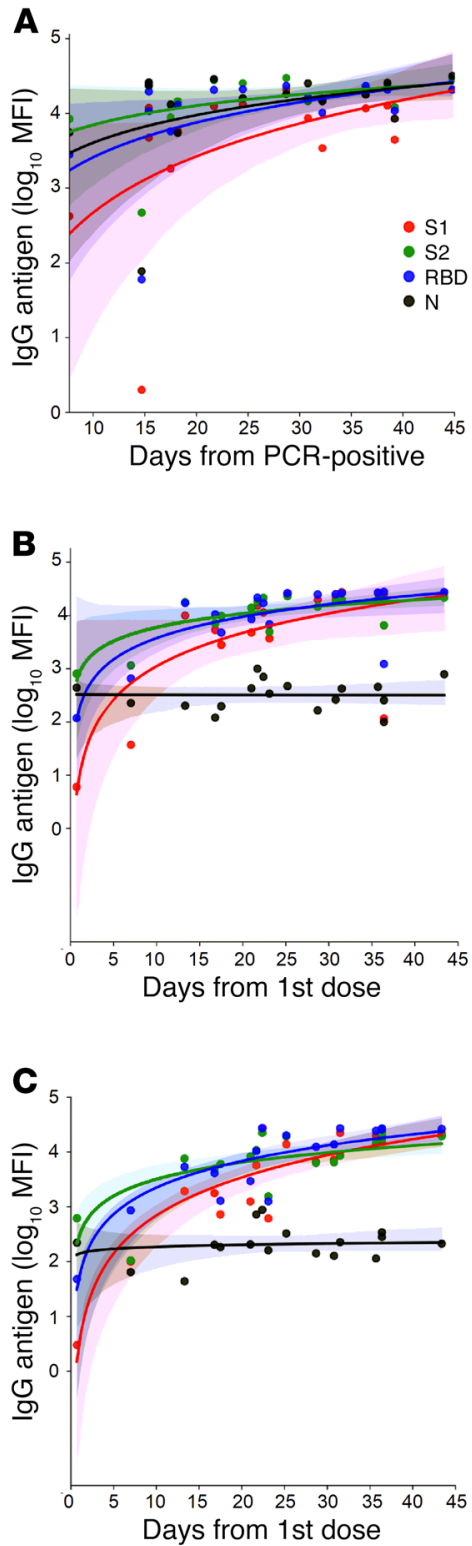

D
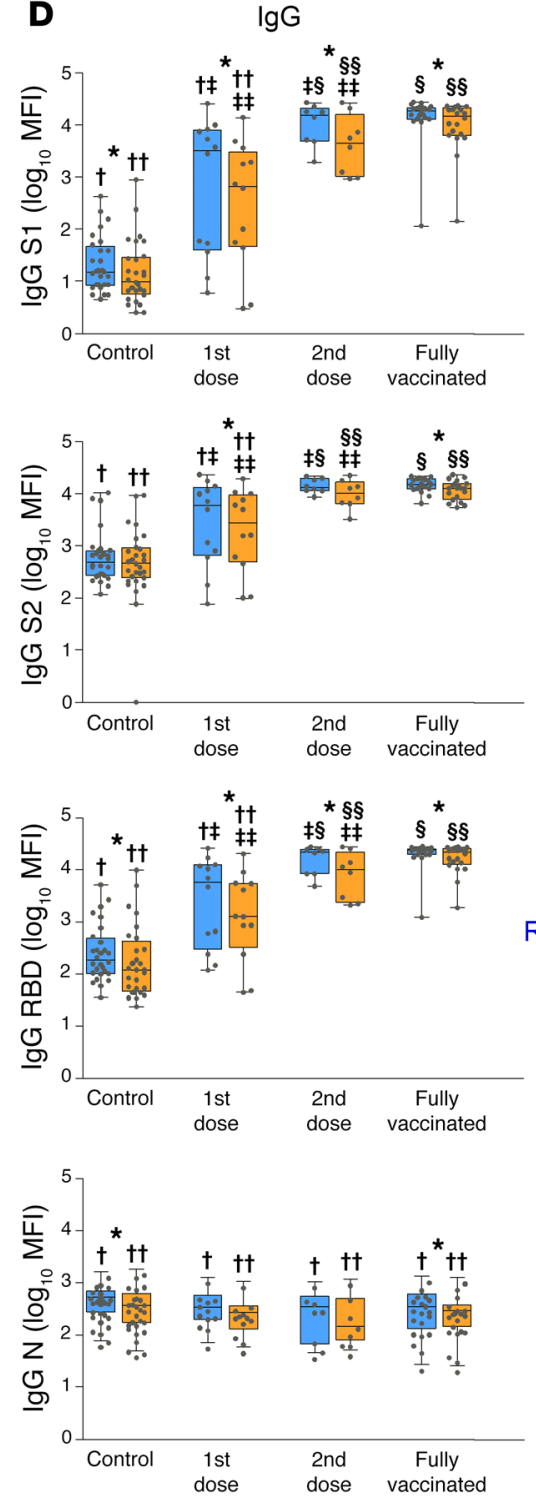
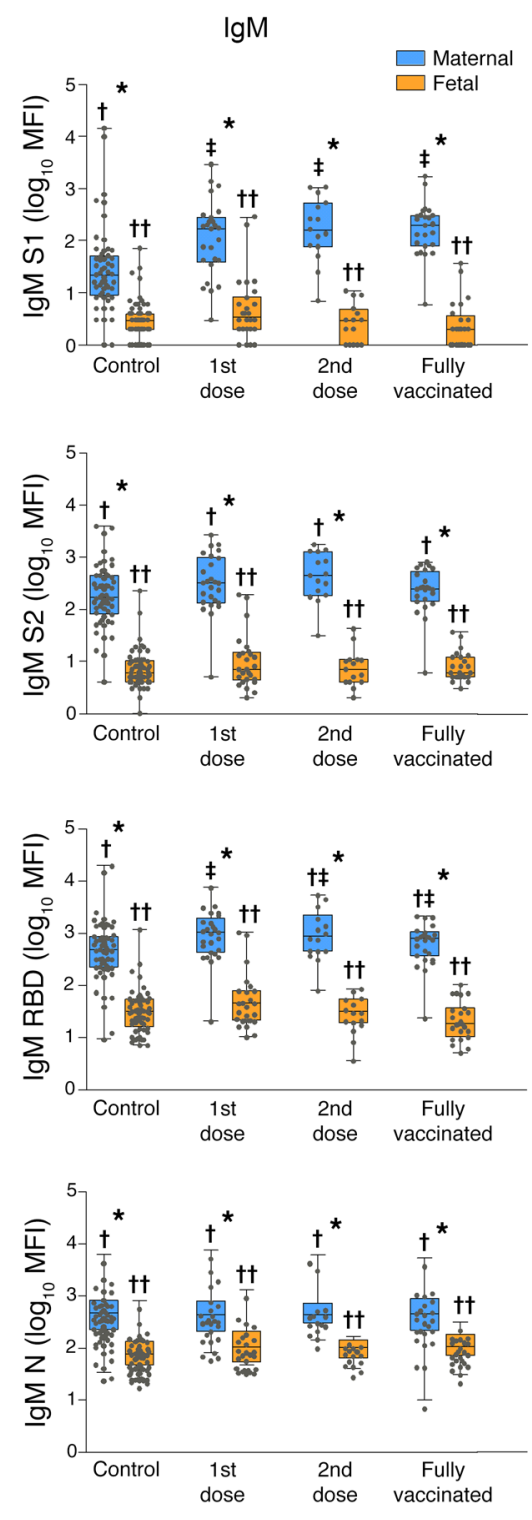

Figure 3. Dependence of immune response to SARS-CoV-2 infection and to vaccination on the duration from exposure to delivery. (A) Analysis of the level of maternal IgG at delivery for acute ( $<50$ days) infection. Time was derived from the GA of RT-PCR positivity and the GA of delivery (see the immune response across pregnancy in Figure 1). (B) Analysis of maternal IgG response to BNT162b2 vaccination derived from the GA of the first vaccine and the GA of delivery. (C) Analysis of the level of fetal IgG at delivery, following BNT162b2 vaccination. Time was derived from the GA of the first vaccine and the GA of delivery. (B and C) A second dose was administered on day 21. Shaded areas and lines in A-C show the mean and $95 \%$ confidence interval. (D) Serological data of maternal-fetal pairs were derived from control, unvaccinated serologically negative $\left(\mathrm{N}^{-}\right)$mothers; as well as vaccinated mothers grouped for deliveries in the first 3 weeks after the first vaccine; deliveries during the first week after the second vaccine; and fully vaccinated who delivered more than a week after the second vaccine. Left columns, IgG; right columns, IgM; from top to bottom, serological response to S1, S2, RBD, and N. Statistical significance: $\uparrow, \ddagger, \S$ above the blue bars indicate significant differences among the groups in maternal antibodies, while $\dagger \dagger, \neq \ddagger, \S \S$ above the orange bars indicate significant differences among the groups in fetal antibodies (Kruskal-Wallis 1-way ANOVA test, following by Dunn's all-pairwise comparisons test). An asterisk indicates a significant difference between maternal and fetal antibodies within the same group (paired $t$ test). Box and whiskers: middle line, median; box, the $25 \%$ and $75 \%$ (25th and 75 th percentiles); whiskers, minimum \& maximum values (Supplemental Table 2).

COVID-19 mRNA vaccine elicits a rapid rise in IgG titers and effective transfer across the placenta, exceeding the TR observed in pregnant women with third trimester SARS-CoV-2 infection, as was previously described in nonpregnant populations and in a small pilot study in pregnancy (25).

Importantly, maternal IgG humoral response to vaccination in noninfected patients readily transfers across the placenta to the fetus, leading to a substantial and potentially protective antiSARS-CoV-2 titer in the neonatal bloodstream, already 2 weeks following the first vaccine dose. Hence, our data deliver convincing proof for the potency of COVID-19 mRNA vaccines to induce robust humoral maternal and neonatal immunity during pregnancy. In addition to transplacental acquired humoral defense, other investigators have recently shown that vaccine response 

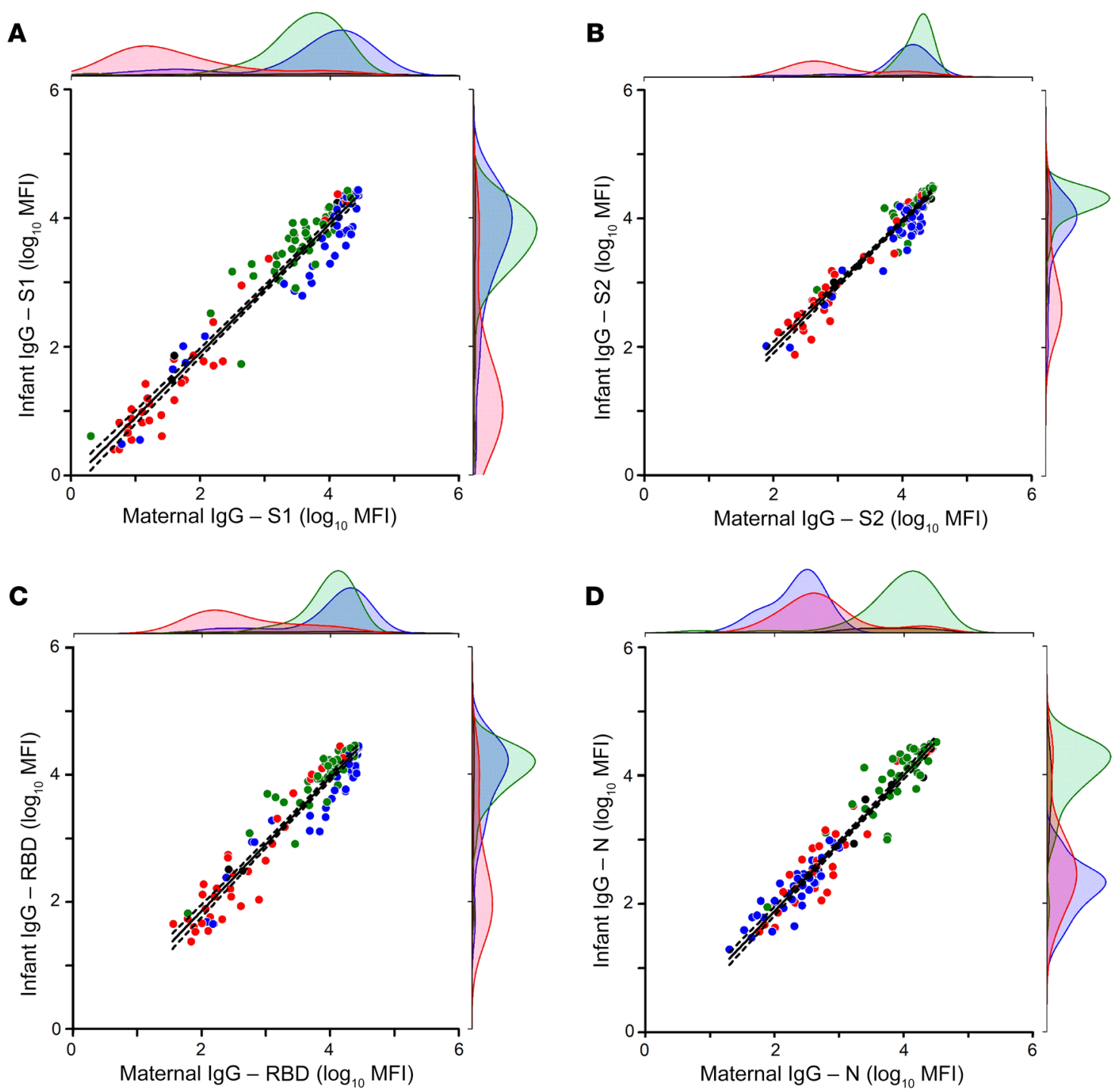

Figure 4. Maternal-fetal serological correlation of IgG for S1, S2, RBD, and N. A subgroup of the control group was identified as serologically positive for $\mathrm{N}$ as well as for S1, S2, and RBD ( $n=9$ of 62). Similarly, a subgroup of the vaccinated was serologically identified as positive for $\mathrm{N}(n=7$ of 86 ; marked in black). A significant maternal-fetal correlation was observed for all groups and all antigens. Correlations between fetal and maternal Ab (N, S1, S2, RBD) were analyzed by LR test (Supplemental Figure 3). Each dot represents data from a single patient; the LR line is marked in black, with its $95 \% \mathrm{Cl}$ (dotted lines). (A) $R^{2}=0.9443$; adjusted $R^{2}=0.9438 ; P<0.0001$. (B) $R^{2}=0.9353$; adjusted $R^{2}=0.9348 ; P<0.0001$. (C) $R^{2}=0.9200$; adjusted $R^{2}=0.9194 ; P<$ 0.0001 . (D) $R^{2}=0.9366$; adjusted $R^{2}=0.9361 ; P<0.0001$. Red, control; green, PCR positive; blue, vaccinated $\mathrm{N}^{-}$; black, vaccinated $\mathrm{N}^{+}$.

included the transfer of both Spike-specific IgG and IgA antibodies into the maternal breastmilk, potentially building another line of defense for breastfed infants (25). Accordingly, antenatal immunization will potentially provide adequate maternal and neonatal protection at highly vulnerable life stages. Nevertheless, sound evidence regarding safety is still needed and should be addressed in future studies.

Using multiplexed serology, we were able to distinguish between viral and vaccination-induced immunity and uncover clusters of asymptomatic, undiagnosed infections among the control and vaccinated groups. We describe 7 vaccinated patients who were found to have high levels of anti-N IgG, corresponding with previous undiagnosed infection. We detected no significant changes in maternal or neonatal titers when compared with the titers of vaccinated and recovered participants. In addition, among the 65 PCR-positive deliveries, we found 5 fetuses (7\%) who showed IgM reactivity to all or most viral antigens, consistent with placenta barrier defect, fetal exposure to viral antigens, or vertical viral transmission. In contrast, among the 86 vaccinated deliveries, we found no evidence for cases of fetal IgM response to any of the vaccine-induced antigens.

Strengths and limitations. The present study has several strengths and limitations. Its strengths include its multicenter design and patient accrual, our relatively large cohort size, and our diverse patient population. Its limitations include the bias in sample collection, as most of the study recruitment occurred during the day, and therefore does not include many of the emergency cases. However, the method of sample collection did not differ between the study groups or medical centers, thus minimizing the impact of this effect on our results. Second, since sample collection began long before 

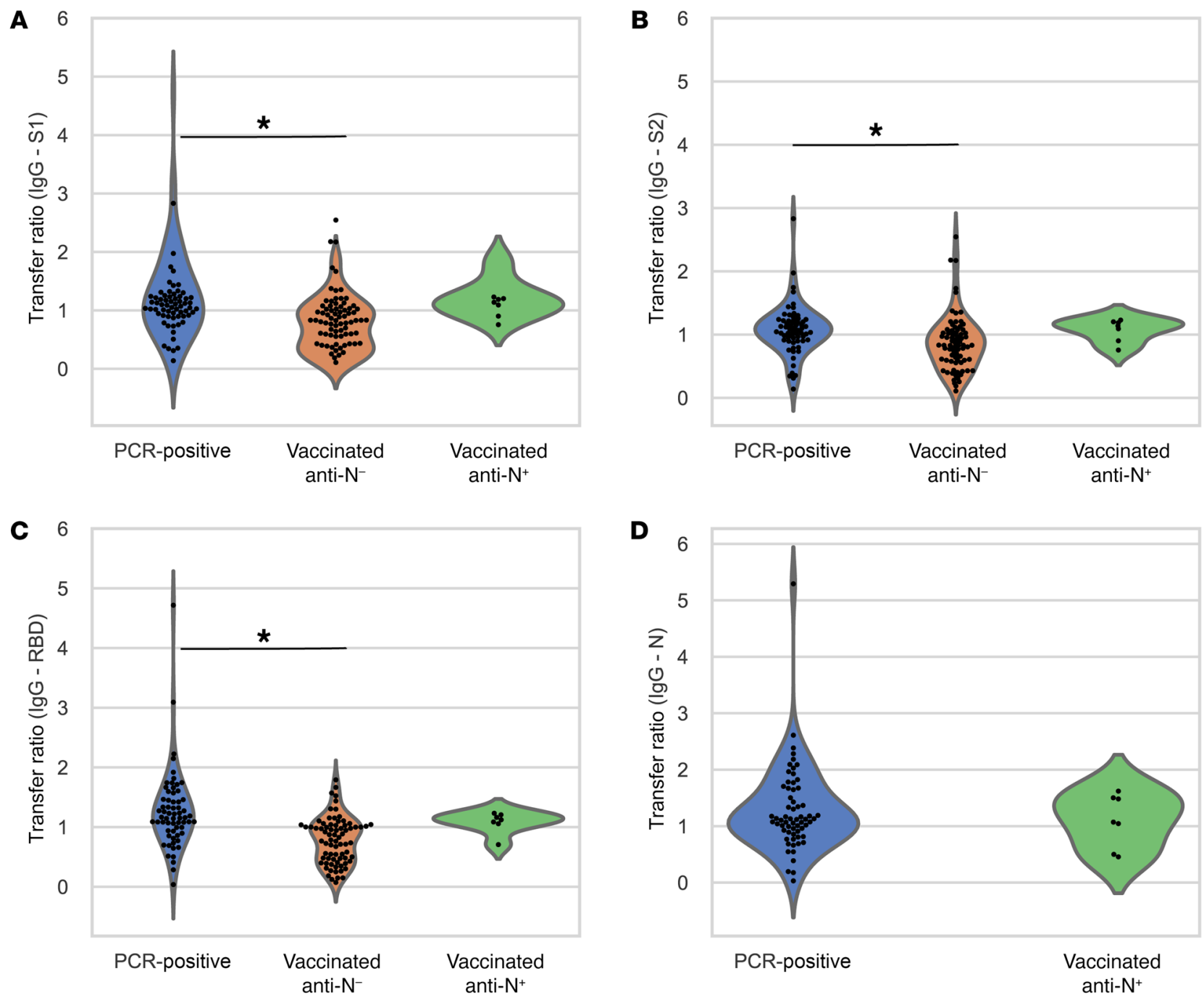

Figure 5. Maternal to fetal transfer ratio for the PCR-positive recovered group and for serologically positive and negative vaccinated groups. Transfer ratios for IgG-S1 (A), IgG-S2 (B), IgG-RBD (C), and IgG-N (D). Note that the TR for $\mathrm{N}$ in the $\mathrm{N}^{-}$group is not presented due to the low seropositivity. Differences among the groups were analyzed by using Kruskal-Wallis 1-way ANOVA test, following by Dunn's all-pairwise comparisons test. For S1, S2, and RBD, there was a significant difference between the PCR-positive vaccinated anti- $\mathrm{N}^{-}$groups $(P<0.0002)$. The transfer ratios did not differ between the vaccinated anti- $\mathrm{N}^{+}$and all the other groups for all antigens $(P=0.4577)$.

the COVID-19 immunization campaign, the duration of sample collection differed between the groups, with an extended recruitment period for COVID-19-recovered cases. We found no differences in background or demographic parameters among the groups. Third, a history of COVID-19 infection during pregnancy was made by positive RT-PCR results during pregnancy with self-reporting of the time of PCR test. Stricter and more accurate symptom monitoring and repeated sampling during pregnancy may provide a higher resolution delineation of how the immune response develops and transfers following COVID-19 infection. Fourth, patients presenting with RT-PCR-positive results within one week prior to delivery were excluded from this study due to safety concerns. Thus, future studies are needed in order to characterize the maternal humoral response to COVID-19 within days of infection.

Conclusions. We show herein a robust maternal humoral immune response coupled to a rise in protective antibodies in the fetal circulation as early as 15 days after the first BNT162b2 mRNA vaccination. We further show that mid-pregnancy SARS-CoV-2 infection results in prolonged maternal and fetal humoral immunity presented at delivery time.

\section{Methods}

Study design. Pregnant women admitted for delivery at 8 medical centers in Israel (Hadassah Mount Scopus, Wolfson, HaEmek, Hillel Yafe, Rabin, Shaare Zedek, Meir, and Sourasky medical centers) were approached for enrollment in a biorepository study, starting in April 2020. Eligibility criteria included an age of 18 years or older and a willingness to participate and provide informed consent. Pregnant women with active maternal COVID-19 disease at delivery were excluded from the study. Eligible patients were identified by dedicated study clinicians (obstetrician, nurse midwife) present on the labor and delivery units enrolled in the study. Gravidae who received the BNT162b2 mRNA vaccine during pregnancy were assigned to the vaccinated group; parturients with documented COVID-19 infection during preg- 
nancy, confirmed by positive nasopharyngeal swab RT-PCR test, comprised the COVID-19 positive group. Unvaccinated parturients were matched to the vaccinated group participants based on clinical parameters (Table 1) and were assigned to a control group.

Sample collection and handling. Maternal and fetal blood samples were collected from the enrolled patients prior to delivery and from the umbilical cord following delivery. The umbilical cord was wiped clean and blood was drawn from the vein. Blood samples were centrifuged at $1000 \mathrm{~g}$ for 10 minutes at room temperature, and serum samples were aliquoted into dedicated precoded tubes and stored at $-80^{\circ} \mathrm{C}$ until analyses at the Weizmann Institute. Fourteen placental tissue samples were microscopically blindly examined by a single experienced pathologist. The rate of malperfusion lesions was similar in the examined placental tissue of all groups (Supplemental Table 2 and Supplemental Figure 4).

Quantification of anti-COVID-19 antibodies. Serum samples were thawed, heat-inactivated at $56^{\circ} \mathrm{C}$ for 30 minutes, and transferred to bar-coded 96-well plates for analysis. Serum IgG and IgM were detected using Milliplex MAP SARS-CoV-2 Antigen Panel 1 IgG (HC12SERG-85K) and IgM (HC19SERM1-85K). Reagents were prepared according to manufacturer instructions and dispensed to 96-well source plates (Greiner 651201, Sigma-Aldrich). Serum samples were diluted 1:100 in assay buffer and added to antigen-immobilized Milliplex beads in 96-well plates using a Bravo liquid handler (Agilent). Plates were covered, shaken for 2 hours at room temperature, and washed 3 times with wash buffer, using a manual magnet and multidrop combi dispenser (Thermo Fisher Scientific). Anti-IgG$\mathrm{PE}$ or anti-IgM-PE conjugate was added, and the samples were incubated (90 minutes with shaking) and washed. Sheath fluid was added to the samples, and net fluorescence intensity (MFI) signals were detected on a Luminex MAGPIX reader (Supplemental Data). Repeat measurements of the same sample showed less than $5 \%$ difference for all antibodies. Positive and negative controls were included in each analysis for accuracy and reproducibility.

Statistics. Statistical analyses were performed using Statistix 8 software (Analytical Software) and Prism 5.01 (GraphPad Software). IgG and IgM antibody (S1, S2, RBD, N) concentrations were $\log _{10}$-transformed for analyses. Correlations between fetal and maternal Ab were analyzed by linear regression test. Comparisons of antibody concentrations among groups, as well as continuous parameters (e.g., clinical data), were analyzed by Kruskal-Wallis 1-way ANOVA test, followed by Dunn's all-pairwise comparisons test; or alternatively, by Wilcoxon rank sum test (if only 2 groups were compared). Comparisons between maternal and fetal concentrations within each group were analyzed by paired $t$ test. Pearson $\chi^{2}$ analysis was used to compare proportional data. All statistical tests were based on 2-tailed hypotheses. Differences were considered significant at $P$ less than 0.05 .

Study approval. The current study followed the Strengthening the Reporting of Observational Studies in Epidemiology (STROBE) reporting guideline. The study was approved by the institutional review boards of all participating medical centers and by the Weizmann Institute of Science. All research participants provided written informed consent prior to enrollment.

\section{Author contributions}

OB, RPM, ZS, AM, HB, SY, MN, and MK designed the research studies. KNS, YSC, RC, RGT, EH, RGB, YJM, TBS, GSM, SFG, HYS, HBR, NDS, DGW, and HB conducted the experiments and collected the samples.RPM, LS, and HB acquired the data. RPM, TR, LS, ZS, HB, SY, MN, and MK analyzed the data. ZS provided reagents. OB, RPM, TR, TBS, DGW, AM, HB, SY, MN, and MK participated in writing the manuscript.

\section{Acknowledgments}

This work was supported by an Israeli Science Foundation KillCorona grant 3777/19 (to MN, MK, SY, AM) and by a research grant from the Weizmann Institute Fondazione Henry Krenter (to MN). We would like to thank the patients who made this research possible. We acknowledge the contribution in patient recruitment, sample preparation and discussions by Gila Meir, Leonardo Solmesky, and Nava Dekel (Weizmann Institute); Adva Cahen Peretz, Michal Lipschuetz, Nadine Souri, and Sarah M. Cohen (Hadassah Medical Center); Yasmin Farhadian and Hind Odeh (Wolfson Medical Center); Shalva Fux (HaEmek Medical Center); Alina Wiener and Luchilla Zorzetti (Hillel Yaffe Medical Center); Itamar Glick (Shaare Zedek Medical Center); and Avital Diamond and Yaara Hoffman (Meir Medical Center).

Address correspondence to: Simcha Yagel, Department of Obstetrics and Gynecology, Hadassah-Hebrew University Medical Center, Jerusalem, Israel. Phone: 972.50.7874478; Email: syagel@ hadassah.org.il. Or to: Michal Neeman, Department of Biological Regulation, The Weizmann Institute of Science, Rehovot 76100, Israel. Phone: 972.8.9342487; Email: michal.neeman@ weizmann.ac.il. Or to: Michal Kovo, Department Obstetrics and Gynecology, Wolfson Medical Center, Holon; affiliated to Sackler Faculty of Medicine, Tel Aviv University, Tel Aviv, Israel. Phone: 972.50.8533119; Email: kobo@wmc.gov.il.
1. DeBolt CA, et al. Pregnant women with severe or critical coronavirus disease 2019 have increased composite morbidity compared with nonpregnant matched controls. Am JObstet Gynecol. 2021;224(5):510.e1-510.e12.

2. Hantoushzadeh S, et al. Maternal death due to COVID-19. Am JObstet Gynecol. 2020;223(1):109-109.

3. Pierce-Williams RAM, et al. Clinical course of severe and critical coronavirus disease 2019 in hospitalized pregnancies: a United States cohort study. Am JObstet Gynecol MFM. 2020;2(3):100134.
4. Juan J, et al. Effect of coronavirus disease 2019 (COVID-19) on maternal, perinatal and neonatal outcome: systematic review. Ultrasound Obstet Gynecol. 2020;56(1):15-27.

5. Dashraath P, et al. Coronavirus disease 2019 (COVID-19) pandemic and pregnancy. Am J Obstet Gynecol. 2020;222(6):521-531.

6. Knight M, et al. Characteristics and outcomes of pregnant women admitted to hospital with confirmed SARS-CoV-2 infection in UK: national population based cohort study. BMJ. 2020;369:m2107.

7. Panagiotakopoulos L, et al. SARS-CoV-2 infec- tion among hospitalized pregnant women: reasons for admission and pregnancy characteristics - eight U.S. Health care centers, March 1-May 30, 2020. MMWR Morb Mortal Wkly Rep. 2020;69(38):1355-1359.

8. Delahoy MJ, et al. Characteristics and maternal and birth outcomes of hospitalized pregnant women with laboratory-confirmed COVID19 - COVID-NET, 13 states, March 1-August 22, 2020. MMWR Morb Mortal Wkly Rep. 2020;69(38):1347-1354.

9. Jering KS, et al. Clinical characteristics and outcomes of hospitalized women giving birth 
with and without COVID-19. JAMA Intern Med. 2021;181(5):714-717.

10. Stafford IA, et al. The coronavirus disease 2019 vaccine in pregnancy: risks, benefits, and recommendations. Am JObstet Gynecol. 2021;224(5):484-495.

11. Centers for Disease Control and Prevention. Data on COVID-19 during pregnancy: severity of maternal illness. https://covid.cdc.gov/coviddata-tracker/?CDC_AA_refVal=https\% 3A\%2F\%2Fwww.cdc.gov\%2Fcoronavirus\% 2F2019-ncov\%2Fcases-updates\%2Fspecialpopulations\%2Fpregnancy-data-on-covid-19. html\#pregnant-population. Updated May 17, 2021. Accessed March 30, 2021.

12. ACOG. Vaccinating pregnant and lactating patients against COVID-19. https://www.acog. org/clinical/clinical-guidance/practice-advisory/ articles/2020/12/vaccinating-pregnant-andlactating-patients-against-covid-19. Updated April 28, 2021. Accessed March 30, 2021.

13. Centers for Disease Control and Prevention. COVID-19 (coronavirus disease): people with certain medical conditions. https://www.cdc. gov/coronavirus/2019-ncov/need-extra- precautions/people-with-medical-conditions. html. Updated May 13, 2021. Accessed March 30, 2021.

14. Goncalves G, et al. Transplacental transfer of measles and total IgG. Epidemiol Infect. 1999;122(2):273-279.

15. Munoz FM, et al. Safety and immunogenicity of tetanus diphtheria and acellular pertussis (Tdap) immunization during pregnancy in mothers and infants: a randomized clinical trial. JAMA. 2014;311(17):1760-1769.

16. Martinez DR, et al. Fc characteristics mediate selective placental transfer of IgG in HIVinfected women. Cell. 2019;178(1):190-201.

17. Atyeo C, et al. Compromised SARS-CoV2-specific placental antibody transfer. Cell. 2021;184(3):628-642.

18. Edlow AG, et al. Assessment of maternal and neonatal SARS-CoV-2 viral load, transplacental antibody transfer, and placental pathology in pregnancies during the COVID-19 pandemic. JAMA Netw Open. 2020;3(12):e2030455.

19. Kim L, et al. Hospitalization rates and characteristics of children aged $<18$ years hospitalized with laboratory-confirmed COVID-19-
COVID-NET, 14 States, March 1-July 25, 2020. Mmwr-Morbidity and Mortality Weekly Report. 2020;69(32):1081-1088.

20. Dong $\mathrm{L}$, et al. Possible vertical transmission of SARS-CoV-2 from an infected mother to her newborn. JAMA. 2020;323(18):1846-1848.

21. Skowronski DM, De Serres G. Safety and efficacy of the BNT162b2 mRNA Covid-19 vaccine. NEngl JMed. 2021;384(16):1576-1577.

22. Baden LR, et al. Efficacy and safety of the mRNA-1273 SARS-CoV-2 vaccine. $N$ Engl J Med. 2021;384(5):403-416.

23. Krammer F, et al. Antibody responses in seropositive persons after a single dose of SARS-CoV-2 mRNA vaccine. $N$ Engl J Med. 2021;384(14):1372-1374.

24. Stamatatos $\mathrm{L}$, et al. mRNA vaccination boosts cross-variant neutralizing antibodies elicited by SARS-CoV-2 infection [published online March 25, 2021]. Science. https://doi.org/10.1126/ science.abg 9175 .

25. Gray KJ, et al. COVID-19 vaccine response in pregnant and lactating women: a cohort study [preprint]. https://doi.org/10.1101/2021.03.07.21 253094. Posted on medRxiv, March 8, 2021. 